\title{
Randomized controlled trial of aquatic exercise for treatment of knee osteoarthritis in elderly people
}

\author{
SIROUS AZIZI ${ }^{1}$, AFSANEH DADARKHAH ${ }^{1}$, ZAHRA REZASOLTANI ${ }^{1}$, \\ SEYED AHMAD RAEISSADAT ${ }^{2,3}$, REZA KAZEMPOOR MOFRAD ${ }^{4}$, SHARIF NAJAFI ${ }^{1, *}$
}

${ }^{1}$ Department of Physical Medicine and Rehabilitation, Faculty of Medicine, Aja University of Medical Sciences, Tehran, Islamic Republic of Iran

${ }^{2}$ Physical Medicine and Rehabilitation Research Center, Shahid Beheshti University of Medical Sciences, Tehran, Islamic Republic of Iran

${ }^{3}$ Shahid Modarres Hospital, Clinical Research and Development Center, Shahid Beheshti University of Medical Sciences,

Tehran, Islamic Republic of Iran

${ }^{4}$ Faculty of Medicine, Shahid Beheshti University of Medical Sciences, Tehran, Islamic Republic of Iran

*Corresponding author: Sharif Najafi; Department of Physical Medicine and Rehabilitation, Faculty of Medicine, Aja University of Medical Sciences, Etemadzadeh St., Western Fatemi, Tehran 14117 18541, Islamic Republic of Iran; Phone: +98 214382 3476; Fax: +98 2188028724 ;

E-mail: sh.najafiajaums.ac.ir

(Received: April 30, 2019; Accepted: June 23, 2019)

\begin{abstract}
Aim: The aim of this study is to assess the efficacy of aquatic exercise on pain, gait, and balance among elderly patients with knee osteoarthritis. Methods: We performed a randomized controlled trial at a university hospital. Overall, 32 men with knee osteoarthritis, aged $\geq 60$ years, were included. Pain, balance, and gait were evaluated before and 2 months after interventions. The group control used acetaminophen and followed lifestyle recommendations. The intervention group performed the aquatic exercise three sessions per week for 8 weeks. Results: At the end of the study, mean pain scores were significantly different between the groups $(p=0.010)$. Within-group analyses showed that group intervention experienced significant pain relief $(p=0.019)$, whereas group control did not show the significant change $(p=0.493)$. There was significant improvement in favor of aquatic exercise with regard to static $(p=0.001)$ and dynamic $(p=0.001)$ balance, step length $(p=0.038)$, stride length $(p<0.001)$, and cadence $(p<0.001)$. However, we did not find a significant difference in step time and width between the two groups. Conclusions: Aquatic exercise would be beneficial in decreasing subjective pain of osteoarthritis. There are some recognizable improvements in patients' gait and balance as well.
\end{abstract}

Keywords: knee osteoarthritis, aquatic exercise, pain, gait, balance

\section{Introduction}

Knee osteoarthritis is a common form of arthritis identified by the physician in daily practice [1]. The prevalence of the disease is about $28 \%$ among the population over the age of 45 years, and people over 63 years are more prone to develop an accelerated form of the disease [2-4]. Patients typically have pain and stiffness in their knees and have a lower quality of life because of reduced physical activity $[5,6]$. Moreover, for patients in the end stage of the disease, surgery is a potential intervention [7]. In addition, psychological distress and socioeconomic burden are still major concerns $[2,8]$.

Symptomatic relief and preventing deterioration of the disease are the main goals of treatment [6]. Various therapeutic and lifestyle interventions might reduce the risk of structural damage and hence the risk of disease activity. Weight reduction plays an important role in reducing the load of the knee joint in overweight patients. Educating patients to use their joints properly would be beneficial to decrease disability and relapse [1]. Analgesic medications such as non-steroidal anti-inflammatory drugs and glucocorticoids are also prescribed frequently to palliate the symptoms and reduce inflammation $[9,10]$. However, central to the recommended treatments is the use of exercise to maintain general health, to recover function, and to decrease the likelihood of the disease progression $[2,11-13]$.

For exercise programs to be successful, patients' adherence is a requirement. Weakness and movement-related

This is an open-access article distributed under the terms of the Creative Commons Attribution-NonCommercial 4.0 International License, which permits unrestricted use, distribution, and reproduction in any medium for non-commercial purposes, provided the original author and source are credited, a link to the CC License is provided, and changes - if any - are indicated. 
pain decrease patients' motivation to complete therapeutic exercise schedule. As an alternative to land exercise, an aquatic exercise in a swimming pool provides a more enjoyable experience for people. Water buoyancy causes the patients to move more comfortably and less painfully than on the land. In addition, warm water provides more muscle relaxation and symptom palliation $[11,14,15]$.

The role of aquatic exercise in the management of knee osteoarthritis has been investigated previously in some studies [16-22]. However, despite growing use, there is a relative paucity of information with regard to the effects of aquatic exercise on balance and gait parameters among elderly people. Some researchers have focused only on changes subjectively perceived within participants with narrow selection criteria $[6,16,23,24]$. Commonly, pain and other joint symptoms, mobility, quality of life, and psychological health have received much attention, whereas the exact pattern and magnitude of the effects on gait and balance over time are still uncertain and controversial [1, 25-29]. Furthermore, evaluation of the results is complicated by the lack of uniformity, limited generalizability to primary care, variation in diagnostic criteria, and poor descriptions of interventions in these studies $[6,16,22$, $23,27,30]$. Therefore, to assess the impact of aquatic exercise on knee osteoarthritis, further studies are required $[20,21]$.

In a recent review study on the effects of aquatic exercise for the treatment of hip and knee osteoarthritis, a small but clinical relevant improvement was reported for pain and disability and quality of life [29]. However, the review showed that lack of highly significant results might be attributable to a low number of studies on aquatic exercise. The study suggested that further research is warranted to optimize aquatic exercise for patients with well-established knee osteoarthritis. In addition, it was recommended that participants should be characterized by age, sex, body mass index, and severity of the disease. Moreover, proposing a control treatment was among the list of suggestions.

The aim of conducting this study was to assess the efficacy of aquatic exercise for the management of knee osteoarthritis among elderly patients. In addition to pain, we particularly paid attention to gait and balance parameters. Our hypothesis was that aquatic exercise would be associated with changes in symptoms, gait, and balance parameters.

\section{Materials and Methods}

\section{Design and setting}

The study was a single-blind randomized controlled trial. The trial was conducted from September 2016 through
May 2017 in an outpatient clinic of the Department of Physical Medicine and Rehabilitation of a University of Medical Sciences.

\section{Recruitment}

We recruited patients from the university hospital. Consecutive male patients aged $\leq 60$ years with an established diagnosis of knee osteoarthritis were enrolled.

The recruitment process of this study started with the invitation of patients to the screening visit of the study. Patients were recruited from the waiting list of the hospital or were referred by other physicians. The majority of the referrals to the clinic was from a rehabilitation center. The study was explained to all potential participants during the interview in the first visit. If a patient declined to participate, another was selected and invited in the same way until the needed sample had been recruited. The diagnoses were documented and confirmed by radiography. A total of 47 patients accepted and attended the screening visit. At the screening visit, all patients filled in a standardized questionnaire for their medical history. A general practitioner carried out a physical examination, and a resident of physical medicine and rehabilitation performed musculoskeletal evaluations. Of these, six patients declined to participate before randomization. At the second visit, potential participants underwent detailed assessments by two of the authors for eligibility as well as compliance. A radiologist, blinded to the patient's histories, read the radiographs. The eligible patients who consented were randomized into two groups.

\section{Eligibility criteria}

We eliminated the confounding effect of sex by avoiding mixed sample of men and women [31]. Male patients with knee osteoarthritis were eligible for the study if their age was $\leq 60$ years, if they had knee pain for at least 3 months, and if they were at the second or third functional level based on clinical and radiological data. In addition, patients entered into the study if they desired to participate in exercise programs.

Exclusion criteria were a history of intra-articular injection within the last 3 months; oral anti-inflammatory drugs within the past 1 week; history of surgery on knee joint or major trauma to the lower limb causing fracture; body mass index more than $34 \mathrm{~kg} / \mathrm{m}^{2}$; and pathologies and diseases affecting knee joint, such as osteonecrosis, severe osteoporosis, rheumatoid arthritis, collagen vascular diseases, and goat. Patients were also excluded if they were in the acute phase of the disease, were addicted to narcotics, and had diabetes. We also confined our sample to male patients. 


\section{Outcome measures}

The primary outcome measure was knee pain. We used a $100-\mathrm{mm}$ visual analog scale to measure subjective pain rated on a scale from 0 (no pain) to 100 (most severe pain). Range of motion in abduction, flexion, and external rotation was assessed using goniometry. We performed the measurements before and 2 months after interventions.

In addition, we measured a variety of secondary outcomes. For the assessment of static balance, we used Romberg's test. The patient stands barefoot with open eyes with arms folded across the chest and feet placed heel to toe with a dominant foot in front. At most for $60 \mathrm{~s}$, the patient should maintain the position, and this time (in seconds) is recorded as the result of the test. Then, the test is repeated with closed eyes [32].

For dynamic balance, we used the Balance Error Scoring System. The system includes three stances: double-leg with hands on the hip and feet together; single-leg with standing on the non-dominant leg and hands on hips; and a tandem stance in which dominant foot is placed in front, heel to toe. The patient closes the eyes and carries out stances on a firm, and an on a foam surface. Then, errors are counted during 20-s trials. Errors include opening eyes; lifting hands off hips; stepping, stumbling, or falling out of position; lifting forefoot or heel; abducting the hip by more than $30^{\circ}$; or failing to return to the test position in more than $5 \mathrm{~s}$ [33].

For assessing gait parameters, we used high-speed camera Casio FH20 with the free, open-source software Kinovea 0.8.15 (http://www.kinovea.org) for the image analysis [34]. Step length $(\mathrm{cm})$, width $(\mathrm{cm})$, time $(\mathrm{s})$, stride length $(\mathrm{cm})$, and cadence (step/min) were recorded.

Diagnosis of osteoarthritis was performed based on clinical and radiological findings. Knee deformity, tenderness, crepitus, effusion, and decreased range of motion were recorded. Joint space narrowing, subchondral sclerosis, osteophytosis, and Kellgren-Lawrence grade were also noticed on radiographs [35]. Anthropometric features including age, weight, height, and body mass index were recorded for all participants as well.

\section{Study intervention}

The group intervention was instructed to use acetaminophen if needed and to follow lifestyle recommendations. The exercise program was designed according to practice recommendations of the American Geriatric Society Panel [36]. Participants in the intervention group followed an aquatic exercise program, three sessions per week for 8 weeks, totaling 24 sessions. The sessions were held in a community pool and provided by physiotherapists certified in aquatic physiotherapy. The water depth was $1.2 \mathrm{~m}$ and the temperature was maintained at about
$32{ }^{\circ} \mathrm{C}\left(89^{\circ} \mathrm{F}\right)$. Each session lasted approximately $60 \mathrm{~min}$ including 10-15 min warm-up, 35-min strength training, and 10-min cool-down exercises.

The group control was also instructed to use only acetaminophen if needed and to follow lifestyle recommendations at least for 8 weeks. At the time of randomization, the patients were informed that they were free to withdraw and to start physical therapy or aquatic exercise if they desired. Only one patient in the group control withdrew from the study and started aquatic exercise in the middle of the program. However, the final analysis was performed according to the intentionto-treat principle.

\section{Ethics}

The trial was conducted in full compliance with the Helsinki Declaration. Ethics approval was obtained from the review boards of the University of Medical Sciences. Patients gave signed written informed consent at the screening visit. The trial investigator explains the aims, advantages, and possible side effects of the trial to the eligible patients.

\section{Sample size, randomization, and blinding}

We tried to find if there were large discrepancies in outcomes between the two groups. Sample size calculation was performed with $\mathrm{G}^{*}$ Power 3.1.5. We estimated a sample size of 16 participants in each group based on the ability to detect a large Cohen's standardized effect size of 0.9 , a power of $80 \%$, and two-tailed $p$ value of 0.05 as statistically significant.

To minimize potential recruitment bias, we collected baseline data before randomization of patients to aquatic exercise and control groups. Patients were randomized by block randomization of two or four to the treatment or control group. An independent statistician generated a random sequence with a computer.

Both participants and treatment providers were aware of an individual's allocation status after randomization. However, an independent assessor, who was blinded to the intervention received by the patients, measured the outcomes at the end of the study. All radiographic images were analyzed by the same reader, who was blinded to the identities of the participants.

\section{Statistical analyses}

Descriptive statistics are presented as mean (standard deviation) for continuous variables. The data were tested on normality using Kolmogorov-Smirnov test. We did not find a significant departure from normality 
Azizi et al.

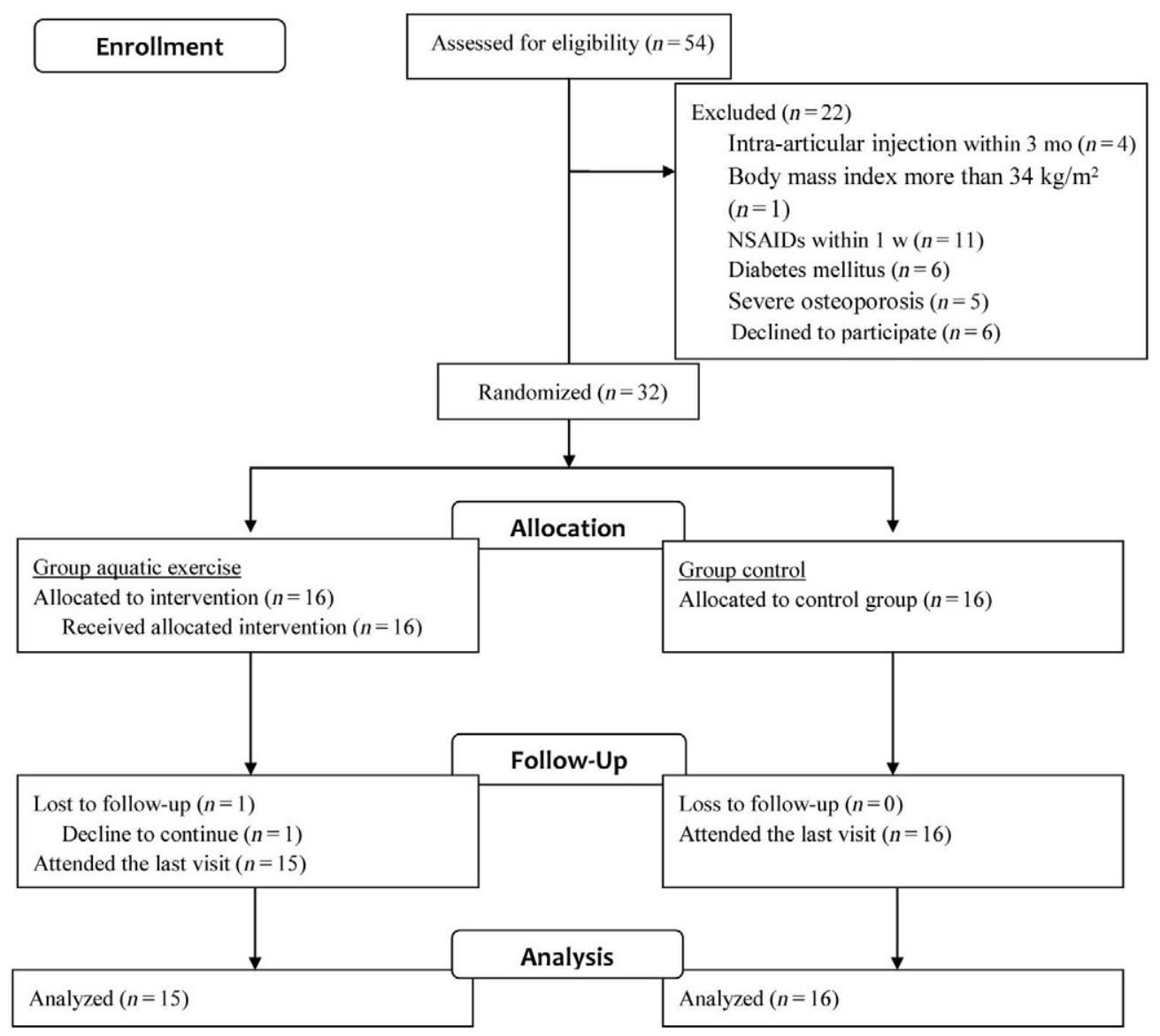

Fig. 1. Patients' flow diagram

in our data. Between-group comparisons were performed using $t$-tests for unpaired data, and withingroup analyses were performed with a paired sample $t$-test. A two-sided $p$ value of 0.05 was used to denote significance. All statistical analyses were carried out using IBM SPSS for Windows, version 21.0 (IBM Corp., Armonk, NY, USA).

\section{Results}

Figure 1 shows the progress of patients through the trial. Overall, 77 patients were invited to participate in and 54 individuals completed both initial study visits. A total of 32 patients were included and randomly allocated to either study arms. Some participants had more than one criterion for exclusion. One patient randomized to group intervention dropped out during the first week after the start of the intervention. Among the 32 patients who were randomly allocated to the groups, 31 (15 in the group intervention and 16 in the group control) completed the study and attended the last assessment visit.

Both groups were similar with regard to mean age, weight, and height (Table I). At the beginning of the study, there was no significant difference in mean visual analog scale for pain between the groups aquatic and control [74.1 (11.5) and 74.2 (24.1), respectively; $p=0.564]$; however, the difference was significant at the end of the study $[64.3$ (19.0) and 74.1 (38.3), respectively; $p=0.010]$. Within-group analyses for mean pain score showed that group intervention experienced significant improvement $(p=0.019)$, whereas group control did not show the significant change $(p=0.493)$. None of the participants reported any serious adverse effect of aquatic exercise.

Table II shows the results of between-group analyses for gait and walking parameters. The results indicated that there was a significant improvement in favor of aquatic exercise with regard to static and dynamic balance, step length, stride length, and cadence. However, we did not

Table I Mean (SD) of anthropometric measurements

\begin{tabular}{lccc}
\multicolumn{4}{c}{ Group } \\
& Aquatic exercise & Control & $p$ value \\
Age (years) & $63.5(4.7)$ & $65.5(3.3)$ & 0.189 \\
Weight $(\mathrm{kg})$ & $75.2(4.2)$ & $78.1(6.4)$ & 0.155 \\
Height $(\mathrm{cm})$ & $178.3(5.4)$ & $176.2(5.8)$ & 0.314 \\
\hline
\end{tabular}


Table II Between-group comparisons of mean for balance and gait parameters

\begin{tabular}{|c|c|c|c|c|}
\hline & \multicolumn{4}{|c|}{ Group } \\
\hline & Assessment & Aquatic exercise & Control & $p$ value \\
\hline Static balance (Romberg's test) & $\begin{array}{l}\text { Baseline } \\
8 \text { weeks }\end{array}$ & $\begin{array}{c}40.5(9.4) \\
54.33(13.4)\end{array}$ & $\begin{array}{l}42.29(9.3) \\
40.16(9.3)\end{array}$ & $\begin{array}{l}0.678 \\
0.001\end{array}$ \\
\hline Dynamic balance (Balance Error Scoring System) & $\begin{array}{l}\text { Baseline } \\
8 \text { weeks }\end{array}$ & $\begin{array}{l}38.3(12.2) \\
30.1(8.2)\end{array}$ & $\begin{array}{r}40.44(12.2) \\
41.1(12.2)\end{array}$ & $\begin{array}{l}0.456 \\
0.001\end{array}$ \\
\hline Step length $(\mathrm{cm})$ & $\begin{array}{l}\text { Baseline } \\
8 \text { weeks }\end{array}$ & $\begin{array}{l}54.9(6.3) \\
60.1(5.2)\end{array}$ & $\begin{array}{l}55.6(6.9) \\
56.2(5.9)\end{array}$ & $\begin{array}{l}0.584 \\
0.038\end{array}$ \\
\hline Step time $(s)$ & $\begin{array}{l}\text { Baseline } \\
8 \text { weeks }\end{array}$ & $\begin{array}{l}0.66(0.04) \\
0.56(0.13)\end{array}$ & $\begin{array}{l}0.67(0.03) \\
0.65(0.11)\end{array}$ & $\begin{array}{l}0.740 \\
0.073\end{array}$ \\
\hline Step width $(\mathrm{cm})$ & $\begin{array}{l}\text { Baseline } \\
8 \text { weeks }\end{array}$ & $\begin{array}{l}11.2(3.8) \\
13.7(4.1)\end{array}$ & $\begin{array}{l}11.7(4.4) \\
11.9(3.4)\end{array}$ & $\begin{array}{l}0.321 \\
0.174\end{array}$ \\
\hline Stride length $(\mathrm{cm})$ & $\begin{array}{l}\text { Baseline } \\
8 \text { weeks }\end{array}$ & $\begin{array}{l}115.1(5.1) \\
133.1(9.4)\end{array}$ & $\begin{array}{l}114.1(4.2) \\
115.1(5.1)\end{array}$ & $\begin{array}{r}0.661 \\
<0.001\end{array}$ \\
\hline Cadence (step/min) & $\begin{array}{l}\text { Baseline } \\
8 \text { weeks }\end{array}$ & $\begin{array}{l}69.3(6.1) \\
95.7(6.9)\end{array}$ & $\begin{array}{l}70.1(6.2) \\
71.4(5.7)\end{array}$ & $\begin{array}{r}0.463 \\
<0.001\end{array}$ \\
\hline
\end{tabular}

find a significant difference in step time and width between the two groups.

\section{Discussion}

We tried to assess the efficacy of aquatic exercise for the management of knee osteoarthritis among male elderly people. We compared pain, gait, and balance between two groups of patients; one underwent aquatic exercise and the other was as the control. Our results suggested that group intervention experienced significant pain decrease compared with group control. In addition, we found that patients in group intervention experienced improvement in their balance and walking. They benefited from aquatic exercise regarding static and dynamic balance, stride and step lengths, and cadence. However, our data failed to show improvement in step time and width because of exercise.

Overall, our results are consistent with the results of some previous studies. In a study, the efficacy of aquatic exercise was compared with patient education in people with knee osteoarthritis [1]. Participants underwent aquatic exercise $(n=31)$ or educational program $(n=29)$ for 8 weeks. Pain, function, quality of life, functional mobility, and depression were assessed before, after 8 weeks and 3 months. The outcomes were in favor of the exercise with regard to pain and function.

In another study, aquatic and land treadmill exercise were compared regarding gait kinematics as well as pain among patients with osteoarthritis. Fourteen patients with knee osteoarthritis aged 43-64 years were included. One of the participants had ankle and another had hip osteoarthritis, too. For 1 week, they performed three exercise sessions at least $24 \mathrm{~h}$ apart for aquatic and land treadmill. Outcomes were assessed before and after each intervention. The study showed that acute training on an aquatic treadmill may be beneficial as a conservative treatment and could favorably influence joint angular velocity and pain in patients with lower limb osteoarthritis. In this study, we also recognized favorable effects on pain and gait for aquatic exercise; however, our research was basically different from that study with regard to design and exercise program. We believe that it will take more than a week to make the differences discernable.

There are few and relatively recent review studies regarding the effects of aquatic exercise. However, they are not similar in their aims, inclusion criteria, results, and interpretation. In a systematic review with meta-analysis, the effects of aquatic exercise on symptoms and lower limb function were investigated [22]. Eleven randomized controlled trials were included. The trials had aquatic exercise group and no-treatment control group. The review indicated that aquatic exercise has favorable effects on pain, self-reported function, and physical functioning. Furthermore, significant effects were observed on stiffness and quality of life. Different outcome measures and small sample sizes of included trials were reported as the limitations of the study. However, it was concluded that aquatic exercise is effective in managing symptoms of lower limb osteoarthritis.

In a systematic review on the effectiveness of aquatic exercise in improving lower limb strength, researchers analyzed data from five large databases (including MEDLINE) [27]. They included 15 randomized controlled trials comparing aquatic exercise containing resistance component with no intervention or land-based exercise, for patients with musculoskeletal conditions. 
The study suggested that the inadequate application of resistance is associated with the limited effectiveness of aquatic exercise in increasing muscle strength. They concluded that future research is needed to recognize the effect of aquatic exercise in improving muscle strength.

In a Cochrane review report, researchers suggested that aquatic exercise may have advantages for the treatment of people with osteoarthritis [29]. They searched for relevant trials up to April 28, 2015 and selected the randomized controlled clinical trials in which aquatic exercise has been compared with a control group for patients with knee or hip osteoarthritis. Usual care and education were among the examples of measures in group control. The number of included trials was 13 and there was a great variation of the included participants. In addition, the exercise lasted for 12 weeks, on average. In general, the study suggested that aquatic exercise has a small favorable effect on the quality of life, pain, and disability. None of the trials reported significant side effect for the treatment. The study listed issues to make further research more consistent. However, regarding gait and balance, there is still a paucity of information. Overall, it seems that the results are still inconclusive and uncertain.

To our knowledge, there was no recent randomized controlled trial comparable to ours with regard to changes in gait and balance variables after a course of aquatic exercise. Our research team was expert, the analyses were straightforward, and the sample was homogenous with regard to sex. The groups were similar at the baseline, and we enjoyed a high participation rate. Meanwhile, we did not investigate clinical outcomes in female patients and did not investigate the effects of aquatic exercise in the long term. Further long-term longitudinal research is warranted to establish the place of aquatic exercise in treating patients with osteoarthritis. Certainly, long-term studies require higher compliance and patience.

\section{Conclusions}

In conclusion, the aquatic exercise would be beneficial for treating knee osteoarthritis at least in the short term. Similar to previous studies, our results indicated that the exercise decreases subjective pain of osteoarthritis. There are some recognizable improvements in patients' gait and balance as well. In general, we prefer to use aquatic exercise alone or combined with other treatment modalities. Our experience showed that patients are more satisfied with the exercise. Minimum adverse effects in addition to increasing the quality of life, reflected in the literature, make aquatic exercise as a suitable option for treatment of osteoarthritis in elderly people.

\section{Highlights}

- Aquatic exercise would be beneficial for treating knee osteoarthritis

- Aquatic exercise is a safe treating for knee osteoarthritis

- Aquatic exercise decreases subjective pain of osteoarthritis

- Aquatic exercise improves patients' gait and balance

- Aquatic exercise would be beneficial for the patient's stride, step lengths, and cadence

$$
* * *
$$

Funding sources: No financial support was received for this study.

Author's contribution: SA contributed to the concept and literature review and coordinated the study. $\mathrm{AD}$ contributed to the design, performed statistical analyses, and interviewed the patients. ZR contributed to the design, guided the development of the study protocol, and participated in physical examination. SAR contributed to the methodology and supervised the study. RKM helped in recruitment, interviewed patients, and supervised exercises. SN contributed to the concept and literature review, coordinated, and supervised the study. All the authors participated in drafting and its final approval.

Conflict of interest: The authors declare no conflict of interest.

\section{References}

1. Taglietti M, Facci LM, Trelha CS, de Melo FC, da Silva DW, Sawczuk G, Ruivo TM, de Souza TB, Sforza C, Cardoso JR: Effectiveness of aquatic exercises compared to patient-education on health status in individuals with knee osteoarthritis: A randomized controlled trial. Clin Rehabil 32, 766-776 (2018)

2. Wellsandt E, Golightly Y: Exercise in the management of knee and hip osteoarthritis. Curr Opin Rheumatol 30, 151-159 (2018)

3. Driban JB, McAlindon TE, Amin M, Price LL, Eaton CB, Davis JE, Lu B, Lo GH, Duryea J, Barbe MF: Risk factors can classify individuals who develop accelerated knee osteoarthritis: Data from the osteoarthritis initiative. J Orthop Res 36, 876-880 (2018)

4. Quintrec JL, Verlhac B, Cadet C, Bréville P, Vetel JM, Gauvain JB, Jeandel C, Maheu E: Physical exercise and weight loss for hip and knee osteoarthritis in very old patients: A systematic review of the literature. Open Rheumatol J 8, 89-95 (2014)

5. Masiero S, Vittadini F, Ferroni C, Bosco A, Serra R, Frigo AC, Frizziero A: The role of thermal balneotherapy in the treatment of obese patient with knee osteoarthritis. Int J Biometeorol 62, 243-252 (2018)

6. Franco MR, Morelhão PK, de Carvalho A, Pinto RZ: Aquatic exercise for the treatment of hip and knee osteoarthritis. Phys Ther 97, 693-697 (2017)

7. Nielsen FK, Egund N, Jørgensen A, Jurik AG: Risk factors for joint replacement in knee osteoarthritis; a 15-year follow-up study. BMC Musculoskelet Disord 18, 510 (2017)

8. Bhatia D, Bejarano $\mathrm{T}$, Novo $\mathrm{M}$ : Current interventions in the management of knee osteoarthritis. J Pharm Bioallied Sci 5, 30-38 (2013)

9. McAlindon TE, Bannuru RR: Osteoarthritis in 2017: Latest advances in the management of knee OA. Nat Rev Rheumatol 14, 73-74 (2018) 
10. da Costa BR, Reichenbach S, Keller N, Nartey L, Wandel S, Jüni P, Trelle S: Effectiveness of non-steroidal anti-inflammatory drugs for the treatment of pain in knee and hip osteoarthritis: A network meta-analysis. Lancet 390, e21-e33 (2017)

11. Rewald S, Mesters I, Lenssen AF, Emans PJ, Wijnen W, de Bie RA: Effect of aqua-cycling on pain and physical functioning compared with usual care in patients with knee osteoarthritis: Study protocol of a randomised controlled trial. BMC Musculoskelet Disord 17, 88 (2016)

12. Munukka M, Waller B, Häkkinen A, Nieminen MT, Lammentausta E, Kujala UM, Paloneva J, Kautiainen H, Kiviranta I, Heinonen A: Physical activity is related with cartilage quality in women with knee osteoarthritis. Med Sci Sports Exerc 49, 1323-1330 (2017)

13. Goh SL, Persson MS, Bhattacharya A, Hall M, Doherty M, Zhang W: Relative efficacy of different types of exercise for treatment of knee and hip osteoarthritis: Protocol for network meta-analysis of randomised controlled trials. Syst Rev 5, 147 (2016)

14. Dias JM, Cisneros L, Dias R, Fritsch C, Gomes W, Pereira L, Santos ML, Ferreira PH: Hydrotherapy improves pain and function in older women with knee osteoarthritis: A randomized controlled trial. Braz J Phys Ther 21, 449-456 (2017)

15. Branco M, Rêgo NN, Silva PH, Archanjo IE, Ribeiro MC, Trevisani VF: Bath thermal waters in the treatment of knee osteoarthritis: A randomized controlled clinical trial. Eur J Phys Rehabil Med 52, 422-430 (2016)

16. Waller B, Munukka M, Rantalainen T, Lammentausta E, Nieminen MT, Kiviranta I, Kautiainen H, Häkkinen A, Kujala UM, Heinonen A: Effects of high intensity resistance aquatic training on body composition and walking speed in women with mild knee osteoarthritis: A 4-month RCT with 12-month follow-up. Osteoarthritis Cartilage 25, 1238-1246 (2017)

17. Munukka M, Waller B, Rantalainen T, Häkkinen A, Nieminen MT, Lammentausta E, Kujala UM, Paloneva J, Sipilä S, Peuna A, Kautiainen $\mathrm{H}$, Selänne $\mathrm{H}$, Kiviranta I, Heinonen A: Efficacy of progressive aquatic resistance training for tibiofemoral cartilage in postmenopausal women with mild knee osteoarthritis: A randomised controlled trial. Osteoarthritis Cartilage 24, 1708-1717 (2016)

18. Milares LP, Assis L, Siqueira A, Claudino V, Domingos H, Almeida $\mathrm{T}$, Tim C, Renno AC: Effectiveness of an aquatic exercise program and low-level laser therapy on articular cartilage in an experimental model of osteoarthritis in rats. Connect Tissue Res 57, 398-407 (2016)

19. Alkatan M, Baker JR, Machin DR, Park W, Akkari AS, Pasha EP, Tanaka H: Improved function and reduced pain after swimming and cycling training in patients with osteoarthritis. J Rheumatol 43, 666-672 (2016)

20. Lu M, Su Y, Zhang Y, Zhang Z, Wang W, He Z, Liu F, Li Y, Liu C, Wang Y, Sheng L, Zhan Z, Wang X, Zheng N: Effectiveness of aquatic exercise for treatment of knee osteoarthritis: Systematic review and meta-analysis. Z Rheumatol 74, 543-552 (2015)

21. Gibson AJ, Shields N: Effects of aquatic therapy and land-based therapy versus land-based therapy alone on range of motion, edema, and function after hip or knee replacement: A systematic review and meta-analysis. Physiother Can 67, 133-141 (2015)
22. Waller B, Ogonowska-Slodownik A, Vitor M, Lambeck J, Daly D, Kujala UM, Heinonen A: Effect of therapeutic aquatic exercise on symptoms and function associated with lower limb osteoarthritis: Systematic review with meta-analysis. Phys Ther 94, 1383-1395 (2014)

23. So BCL, Kong ISY, Lee RKL, Man RWF, Tse WHK, Fong AKW, Tsang WWN: The effect of Ai Chi aquatic therapy on individuals with knee osteoarthritis: A pilot study. J Phys Ther Sci 29, 884-890 (2017)

24. Alcalde GE, Fonseca AC, Bôscoa TF, Gonçalves MR, Bernardo GC, Pianna B, Carnavale BF, Gimenes C, Barrile SR, Arca EA: Effect of aquatic physical therapy on pain perception, functional capacity and quality of life in older people with knee osteoarthritis: Study protocol for a randomized controlled trial. Trials 18, 317 (2017)

25. Wang TJ, Lee SC, Liang SY, Tung HH, Wu SF, Lin YP: Comparing the efficacy of aquatic exercises and land-based exercises for patients with knee osteoarthritis. J Clin Nurs 20, 2609-2622 (2011)

26. Marconcin P, Espanha M, Teles J, Bento P, Campos P, André R, Yázigi F: A randomized controlled trial of a combined selfmanagement and exercise intervention for elderly people with osteoarthritis of the knee: The PLE ${ }^{2} \mathrm{NO}$ program. Clin Rehabil $32,223-232$ (2018)

27. Heywood S, McClelland J, Mentiplay B, Geigle P, Rahmann A, Clark R: Effectiveness of aquatic exercise in improving lower limb strength in musculoskeletal conditions: A systematic review and meta-analysis. Arch Phys Med Rehabil 98, 173-186 (2017)

28. Dannaway J, New CC, New CH, Maher CG: Aquatic exercise for osteoarthritis of the knee or hip (PEDro synthesis). Br J Sports Med 51, 1233-1234 (2017)

29. Bartels EM, Juhl CB, Christensen R, Hagen KB, DanneskioldSamsøe B, Dagfinrud H, Lund H: Aquatic exercise for the treatment of knee and hip osteoarthritis. Cochrane Database Syst Rev 3, CD005523 (2016)

30. Seib C, Anderson D: Can dance-based aquatic exercise improve functionality in obese women with knee osteoarthritis? Menopause 24, 724-725 (2017)

31. Vina ER, Ran D, Ashbeck EL, Ratzlaff C, Kwoh CK: Race, sex, and risk factors in radiographic worsening of knee osteoarthritis. Semin Arthritis Rheum 47, 464-471 (2018)

32. Yim-Chiplis PK, Talbot LA: Defining and measuring balance in adults. Biol Res Nurs 1, 321-331 (2000)

33. Bell DR, Guskiewicz KM, Clark MA, Padua DA: Systematic review of the balance error scoring system. Sports Health 3, 287-295 (2011)

34. Padulo J, Vando S, Chamari K, Chaouachi A, Bagno D, Pizzolato F: Validity of the MarkWiiR for kinematic analysis during walking and running gaits. Biol Sport 32, 53-58 (2015)

35. Braun HJ, Gold GE: Diagnosis of osteoarthritis: imaging. Bone 51, 278-288 (2012)

36. American Geriatrics Society Panel on Exercise and Osteoarthritis: Exercise prescription for older adults with osteoarthritis pain: Consensus practice recommendations. A supplement to the AGS Clinical Practice Guidelines on the management of chronic pain in older adults. J Am Geriatr Soc 49, 808-823 (2001) 